panied by evidence of instabilities with frequencies near the ion cyclotron frequency. Because a mirror reactor requires near-classical confinement this result is not encouraging; it is possible, however, that the necessary quiescence can be achieved by careful machine design and control of the particle velocity distribution.

Theoretical work presented at the conference dealt chiefly with plasma confinement and stability in the Tokamak. There is now a fair measure of agreement on the classical theory of confinement in symmetric low beta systems. Attention is now paid to the effect of residual fiuctuations on such systems and contributions from Dr S. Yoshikawa (Princeton University) and Drs A. A. Galeev and R. Z. Sagdeev (Kurchatov Institute, Moscow) reached similar conclusions by rather different routes. This work emphasizes the role of the poloidal magnetic field in plasma confinement, the much stronger toroidal field performing only an auxiliary function. On the question of stability, the situation is not clear ; a contribution by Drs D. Pfirsch and H. Tasso (Institute for Plasma Physics, Garching) which showed the Tokamak to be unstable against a simple mode will undoubtedly be the subject of much critical attention.

For the first time at these meetings a session was devoted to reactor systems. The broad conclusion from this session was that within the necessarily very wide margins of error, fusion as a power source might be competitive with other systems and might offer substantial environmental advantages.

\section{RNA}

\section{Messenger's Burden}

from our Molecular Biology Correspondent ONE of the strangest molecules that explorers of the eukaryotic nucleus have brought to light is polyadenylic acid, in almost monodisperse form. This observation became more than a mere unexplained curiosity when Lim and Canellakis reported last year that haemoglobin messenger RNA contains a long tract of poly $\mathrm{A}$, which apparently largely accounts for the discrepancy between its observed and expected molecular weight.

The question of how general this phenomenon may be has now been taken up in several laboratories, and a clutch of three articles on the subject is to be found in the current issue of the Proceedings of the US National Academy of Sciences.

Lee, Mendecki and Brawerman (68, 1331 ; 1971) have examined the messenger RNA of ascites cells. They find that at $p \mathrm{H} \mathrm{9,} \mathrm{though} \mathrm{not} \mathrm{under}$ more nearly neutral conditions, a large proportion of the rapidly labelling
RNA fraction of polysomes is specifically trapped on a membrane filter. The fraction so obtained leaves after digestion with pancreatic or $T_{1}$ ribonuclease a sedimentable core consisting largely or wholly of adenylic acid residues, a label in adenosine being almost completely recovered in this resistant fragment, whereas a label in uridine vanishes. Moreover, the behaviour of synthetic poly $A$ on the membrane filters makes it clear that it is the poly $\mathbf{A}$ segment that causes the messenger to be retained. The poly A core recovered from the messenger sediments at about the same rate as tRNA. Another property of the messenger, conferred on it by its poly A, is its tendency, depending on the salt conditions, to enter the wrong phase during phenol extraction.

Very similar results were obtained by Edmonds, Vaughan and Nakazato (ibid., 1336) for the messenger RNA of HeLa cells. The poly A core was shown by polyacrylamide gel electrophoresis to have a very narrow molecular weight distribution, and an esti- mated length of 150-200 nucleotides. This is in fact rather too high to be accounted for by the estimated poly $A$ content of the messenger, but it does not necessarily follow that some of the messenger is devoid of poly $\mathbf{A}$ : a number of other explanations are possible, for example that some of the label goes into other kinds of RNA than messenger.

The chief object of the article, however, is to treat the poly $A$ as an identifying feature, and to look for its presence in the rapidly labelling polydisperse high molecular weight RNA present in the nucleus, which many workers in the field would like to believe is a messenger precursor. Poly $A$ is indeed recovered from this material by digestion, and is of the same size as that found in the messenger. It makes up only some 0.5 per cent of the total. That the poly $\mathrm{A}$ is a part of a large RNA molecule was demonstrated by its failure to dissociate and redistribute itself between labelled and unlabelled nuclear RNA after addition of a high concentration of an organic

\title{
Acceleration of Cosmic Rays
}

THE drawback of the venerable Fermi mechanism for the generation of the cosmic ray spectrum is the large energies that the particles need to have before the mechanism comes into play. Although the scattering of cosmic ray particles by irregularities in the magnetic fields of interstellar space will produce an energy distribution which matches the experimental data on cosmic rays, as Fermi suggested the particles have to have initial energies of 200 $\mathrm{MeV}$ if they are protons, and higher energies if they are heavier particles.

A process which will raise the energies of particles from thermal energies to sufficiently high energies for injection into the Fermi mechanism is suggested in next Monday's number of Nature Physical Science. A group at the University of Bologna argue that particles able to take part in the Fermi mechanism may come from the nucleus of the galaxy, where they have been accelerated from thermal energies by interactions with the magnetic fields of neutron stars.

This notion seems to be inspired by the pulses recorded by Weber's gravitational wave equipment, and whatever the explanation of the data published by Weber, his results have stimulated theoretical work on the conditions in the nuclei of galaxies. Interactions among clusters of collapsed objects at the centre of the galaxy might be a source of gravitational waves, but there are other more prosaic reasons for supposing that the nuclei of galaxies contains dense matter. In any case, the
Bologna group consider a cluster of neutron stars, a proportion of which have strong magnetic fields, and show how charged particles can gain energy by being scattered by the fields.

Protons need to be scattered only twice to gain the $200 \mathrm{MeV}$ that is the entry ticket for the Fermi process. Heavier particles may need to be scattered more than twice, but there is no reason why the $1 \mathrm{BeV}, 20 \mathrm{BeV}$, and $300 \mathrm{BeV}$ that are the required injection energies for alpha particles, oxygen, and iron respectively should not be achieved in this way.

Little more can be said without knowing more about the abundances of the elements in the interstellar gas in the galactic nucleus, and little light is likely to be thrown on this aspect of the problem for many years. The hope is, of course, that the abundances, together with the fact that it becomes progressively more difficult to provide the heavier elements with the necessary injection energy, will provide a match with the element abundances observed from measurements on cosmic rays.

The Bologna article is well in tune with recent trends in astrophysics. The beginnings of a realization of the parts played by high energy processes and by collapsed objects are suggesting several ways in which the acceleration of cosmic rays may be accomplished. For example, particles thrown out of the atmospheres of pulsars by their rapid rotation rates may also have high enough energies to take part in cosmic ray processes. 